International Journal of Pure and Applied Mathematics

Volume 114 No. 4 2017, 719-724

ISSN: 1311-8080 (printed version); ISSN: 1314-3395 (on-line version)

url: http://www.ijpam.eu

doi: $10.12732 /$ ijpam.v114i4.3

\title{
A CLASSIFICATION OF RAMANUJAN COMPLEMENTS OF UNITARY CAYLEY GRAPHS
}

\author{
Reza Safakish ${ }^{1}$, Shahram Mehry ${ }^{2} \S$ \\ ${ }^{1,2}$ Department of Mathematics \\ Faculty of Science \\ Bu-Ali Sina University, Hemedan, IRAN
}

\begin{abstract}
The unitary Cayley graph on $n$ vertices, $X_{n}$, has vertex set $\mathbb{Z}_{n}$, where two vertices $a$ and $b$ are connected by an edge if and only if they differ by a multiplicative unit modulo $n$, i.e. $\operatorname{gcd}(a b, n)=1$. A $k$-regular graph $X$ is Ramanujan if and only if $\lambda(X) \leq 2 \sqrt{k-1}$ where $\lambda(X)$ is the second largest absolute value of the eigenvalues of the adjacency matrix of $X$. We obtain a complete characterization of the cases in which the complements of unitary Cayley graph $\bar{X}_{n}$ is a Ramanujan graph.
\end{abstract}

AMS Subject Classification: $05 \mathrm{C} 25$

Key Words: graph, Ramanujan graphs, unitary Cayley graphs

\section{Introduction}

The eigenvalues of a graph are eigenvalues of its adjacency matrix, and the spectrum of a graph is the collection of its eigenvalues together with multiplicities. If $\lambda_{1}, \lambda_{2}, \ldots, \lambda_{k}$ are distinct eigenvalues of a graph $X$ and $m_{1}, m_{2}, \ldots, m_{k}$ the corresponding multiplicities, then we denote the spectrum of $X$ by

$$
\operatorname{Spec}(X)=\left(\begin{array}{cccc}
\lambda_{1} & \lambda_{2} & \cdots & \lambda_{k} \\
m_{1} & m_{2} & \cdots & m_{k}
\end{array}\right) .
$$

Given a finite group $G$ and a symmetric subset $S$ of $G$, we define the Cayley

Received: $\quad$ July 24,2016

Revised: $\quad$ May 9, 2017

Published: June 7, 2017

$\S_{\text {Correspondence author }}$ (c) 2017 Academic Publications, Ltd. url: www.acadpubl.eu 
graph $X=\operatorname{Cay}(G, S)$ to be the graph whose vertex set is $G$, in which two vertices $v$ and $u$ in $G$ are connected by an edge if and only if $v u^{-1}$ is in $S$. A Cayley graph of the form $\operatorname{Cay}(G, S)$ with $G=\mathbb{Z}_{n}$ is called a circulant graph. It is easy to see that $\operatorname{Cay}(G, S)$ is a simple, $|S|$-regular graph.

The unitary Cayley graph on $n$ vertices, $X_{n}$, is defined to be the undirected graph whose vertex set is $\mathbb{Z}_{n}$, in which two vertices $a$ and $b$ are connected by an edge if and only if $\operatorname{gcd}(a-b, n)=1$. This can also be stated as $X_{n}=$ $\operatorname{Cay}\left(\mathbb{Z}_{n}, \mathbb{U}_{n}\right)$, where $\mathbb{Z}_{n}$ is the additive group of integers modulo $n$ and $U_{n}=\mathbb{Z}_{n}^{*}$ is the set of multiplicative units modulo $n$. $X_{n}$ is a simple, $\varphi(n)$-regular graph, where $\varphi$ is the Euler totient function. When discussing $X_{n}$, we always assume $n>3$

Lemma 1. The eigenvalues of any adjacency matrix of $X_{n}$ are

$$
\lambda_{m}(n)=\mu\left(\frac{n}{(n, m)}\right) \frac{\varphi(n)}{\varphi\left(\frac{n}{(n, m)}\right)},
$$

where $\mu$ is the Mobius function.

Proof. see [4, Klotz, W. and Sander, T. (2007)]

When $\frac{n}{(n, m)}$ is square-free,

$$
\left|\lambda_{m}(n)\right|=\frac{\varphi(n)}{\varphi\left(\frac{n}{(n, m)}\right)} .
$$

\section{Corollary 2.}

$$
\operatorname{Spec}\left(X_{p^{\alpha}}\right)=\left(\begin{array}{ccc}
p^{\alpha}-p^{\alpha-1} & -p^{\alpha-1} & 0 \\
1 & p-1 & p^{\alpha}-1
\end{array}\right) .
$$

The complement $\bar{X}$ of a graph $X$ is the graph with the same vertex set as $X$ such that two vertices are adjacent in $\bar{X}$ if and only if they are not adjacent in $X$.

Lemma 3 ([2]). Let $X$ be an $k$-regular graph with $n$ vertices. Then $X$ and $\bar{X}$ have the same eigenvectors, and their largest eigenvalues are $k$, and $n-k-1$ respectively. Moreover, if the eigenvalues of $X$ are $k, \lambda_{2}, \ldots, \lambda_{n}$, then the eigenvalues of $\bar{X}$ are $n-k-1,1-\lambda_{2}, \ldots, 1-\lambda_{n}$.

Lemma 1 and Corollary2 together imply the following result. 


\section{Corollary 4.}

$$
\operatorname{Spec}\left(\bar{X}_{p^{\alpha}}\right)=\left(\begin{array}{cc}
p^{\alpha-1}-1 & -1 \\
p & p^{\alpha}-p
\end{array}\right)
$$

\section{Ramanujan Unitary Cayley Graphs}

Recall that the adjacency matrix of any $k$-regular graph $X$ has eigenvalues between $k$ and $k$, where $k$ is an eigenvalue with multiplicity precisely equal to the number of connected components of $X$. Furthermore, if $\lambda(X)$ denotes the largest absolute value of the eigenvalues of the adjacency matrix of $X$, smaller than $k$, then the graph $X$ is called Ramanujan if and only if

$$
\lambda(X) \leq 2 \sqrt{k-1}
$$

Note that $\lambda(X)$ is only defined for regular graphs $X$ with 3 or more vertices. Writing $n$ in the form $p_{1}^{\alpha_{1}} p_{2}^{\alpha_{2}} \cdots p_{k}^{\alpha_{k}}$ for some distinct primes $p_{1}<p_{2}<\cdots<p_{k}$, and positive integers $\alpha_{1}, \ldots, \alpha_{k}$, we can determine $\lambda\left(X_{n}\right)$ as follows. Since $X_{n}$ is $\varphi(n)$-regular, we find the maximum absolute value of an eigenvalue $\lambda_{m}(n)$ of the adjacency matrix of $X_{n}$, smaller than $\varphi(n)$. This can be accomplished by looking at 1 . Indeed, we see that if $n=2^{\alpha}$; then the eigenvalues have absolute value of either 0 or $\varphi(n)$ (since, the only values of $m, 0 \leq m \leq n-1$, which make $\frac{n}{(n, m)}$ square-free are $m=0$ and $\left.m=2^{\alpha-1}\right)$, resulting in eigenvalues $\varphi(n)$ and $-\varphi(n)$. Thus $\lambda\left(X_{2^{\alpha}}\right)=0$ and so $X_{2^{\alpha}}$ satisfies 2 and thus is Ramanujan. It is known from [1] that the graph $X_{n}$ is Ramanujan if and only if $n$ satisfies one of the following conditions for some distinct odd primes $p<q$ and natural $\alpha$.

1. $n=2^{\alpha}, \alpha \geq 2$.

2. $n=p$.

3. $n=2^{\alpha} p$ with $\alpha \geq 1, p>2^{\alpha-3}+1$.

4. $n=p^{2}, 2 p^{2}, 4 p^{2}$.

5. $n=p, 2 p q$ with $p<q \leq 4 p-5$.

6. $n=4 p q$ with $p<q \leq 2 p-3$. 


\section{Ramanujan Complements of Unitary Cayley Graphs}

Lemma $5([3])$. Let $p_{1}^{\alpha_{1}} p_{2}^{\alpha_{2}} \cdots p_{k}^{\alpha_{k}}$, be the canonical factorization of an integer $n$ into prime powers, where $p_{1}<p_{2}<\cdots<p_{k}$ are primes and each $\alpha_{i} \geq 1$. If $k \geq 3$ or $k=2$ and $p_{1}>2$, then $2^{k-1} \varphi(n)>n$.

Theorem 6. Let $n \geq 2$ be a intager. Then $\bar{X}_{n}$ is Ramanujan if and only if $n$ is one of the following forms:

1. $n=p^{a}$ with $p$ a prime and $a \geq 1$

2. $n=p^{a} q^{b}$ with $p=2, q=3$ and $1 \leq a \leq 3, b=1$, or $a=1,1 \leq b \leq 2$.

3. $n=p q$ with $p$ and $q$ are primes and $3 \leq p \leq 5,5 \leq q \leq 7$.

4. $n=2 \cdot 5,2 \cdot 3 \cdot 5$

Proof. By (4), $\bar{X}_{p^{a}}$ is Ramanujan.

Case 1: $p_{1}=2$ and $k \geq 2$. In this case, $\lambda\left(\bar{X}_{n}\right)=\varphi(n)-1$ and $\bar{X}_{n}$ is Ramanujan if and only if

$$
\varphi(n)-1 \leq 2 \sqrt{n-\varphi(n)-2}
$$

This condition is satisfied if only $\varphi(n)^{2}<4 n$. In particular, if $k \geq 4$, then by Lemma $5, \varphi(n)^{2}>4 n$ and so $\bar{X}_{n}$ is not Ramanujan. Assume $k \leq 3$.

Case 1.1: $k=3$ and $n=2^{a} p^{b} q^{c}$. Since $(p-1)^{2}=p(p-2)+1>p(p-2)$, If $a \geq 3$, then

$$
\frac{\varphi(n)^{2}}{n}=2^{a-2} p^{b-1} q^{c-1} \frac{(p-1)^{2}}{p} \frac{(q-1)^{2}}{q}>2(p-2)(q-2)>4
$$

and so $\bar{X}_{n}$ is not Ramanujan. It is easy to see that if $b \geq 2$ or $c \geq 2$, then $\varphi(n)^{2}>4 n$, and so $\bar{X}_{n}$ is not Ramanujan. It remains to consider the case where $n=2 p q$ or $4 p q$. If $n=2 p q$, then

$$
\frac{\varphi(n)^{2}}{n}=\frac{(p-1)^{2}(q-1)^{2}}{2 p q}>\frac{(p-2)(q-2)}{2}
$$

If $p \neq 3$ or $p=3$ and $q \geq 7$, then $\varphi(n)^{2}>4 n$, and so $\bar{X}_{n}$ is not Ramanujan. It is easy to see that $n=2 \cdot 3 \cdot 5$, then $\bar{X}_{n}$ is Ramanujan, whilst if $n=2 \cdot 3 \cdot 7$, $\bar{X}_{n}$ is not Ramanujan. If $n=4 p q$, similar to earlier state if $p \neq 3$, or $p=3$ and $q \neq 5$, then $\bar{X}_{n}$ is not Ramanujan. For $n=4 \cdot 3 \cdot 5$, the condition (6) is not established and so $\bar{X}_{n}$ is not Ramanujan. 
Case 1.2: $k=2$ and $n=2^{a} p^{b}$. In this case

$$
\frac{\varphi(n)^{2}}{n}=\frac{2^{a-1} p^{b-1}}{2 p}(p-1)
$$

As case 1.1 , If $a \geq 4, p \geq 7$ or $b \geq 3$, then $\varphi(n)^{2} \geq 4 n$ and so $\bar{X}_{n}$ is not Ramanujan. It is easy to see that $n=2 \cdot 3,2 \cdot 3^{2}, 2 \cdot 5,2^{2} \cdot 3,2^{3} \cdot 3$; then, $\bar{X}_{n}$ is Ramanujan.

Case 2: $p_{1} \geq 3$. In this case $\lambda\left(\bar{X}_{n}\right)=\frac{\varphi(n)}{p_{1}-1}-1$ and so $\bar{X}_{n}$ is Ramanujan if and only if

$$
\frac{\varphi(n)}{p_{1}-1}-1 \leq 2 \sqrt{n-\varphi(n)-2}
$$

this inequality is equivalent to

$$
\frac{\varphi(n)}{p_{1}-1} \leq-(2 p-3)+\sqrt{4 n-9+(2 p-3)^{2}}
$$

and this condition is not satisfied unless

$$
\varphi(n)^{2} / n<4(p-1)^{2}
$$

If $k \geq 4$, then by Lemma $5, \varphi(n)^{2} / n>\varphi(n) / 2^{k-1}>4(p-1)$ and so $\bar{X}_{n}$ is not Ramanujan. Assume $k \leq 3$ and $n=p^{a} q^{b} r^{c}$.

Case 2.1: $k=3$. In this case, we have

$$
\frac{\varphi(n)^{2}}{n}=p^{a-1} q^{b-1} r^{c-1} \frac{(p-1)^{2}(q-1)^{2}(r-1)^{2}}{p q r} \geq \frac{p^{a-1}}{p}(p-1)^{2}(q-2)(r-2)
$$

Therefore, if $a \geq 3$, then $\varphi(n)^{2} / n \geq 4(p-1)^{2}$ and so $\bar{X}_{n}$ is not Ramanujan. Similarly, if $b \geq 2, c \geq 2$ or $p \geq 7$, or $n=9 p q, 25 p q$, then $\bar{X}_{n}$ is not Ramanujan. It is easy to see that $n=3 \cdot 5 \cdot 7$; then, (8) is violated and again $\bar{X}_{n}$ is not Ramanujan. Moreover, If $n=3 p q$, with $p \neq 3$ and $q \neq 7$, then

$$
\frac{\varphi(n)^{2}}{n}=\frac{4(p-1)^{2}(q-1)^{2}}{3 p q}>(p-2)(q-2) \geq 16=4(3-1)^{2}
$$

and so $\bar{X}_{n}$ is not Ramanujan.

Case 2.2: $k=2$ and $n=p^{a} q^{b}$. In this case by Lemma 5,

$$
\frac{\varphi(n)}{n}>\frac{1}{2} \Rightarrow \frac{\varphi(n)^{2}}{n}>\frac{\varphi(n)}{2}=\frac{p^{a-1} q^{b-1}(p-1)(q-1)}{2}>\frac{p^{a-1} q^{b-1}(p-1)^{2}}{2}
$$


and so if $a \geq 3, b \geq 2$ or $a=b=2$, then (9) is violated and $\bar{X}_{n}$ is not Ramanujan. If $n=p q$, then from (8), we have $(q-2)^{2} \leq 4 p$ and so if $q>7$, then $\bar{X}_{n}$ is not Ramanujan. It is easy to see that $n=3 \cdot 5,3 \cdot 7,5 \cdot 7$, then $\bar{X}_{n}$ is not Ramanujan. If $n=p q^{2}$, then

$$
\frac{\varphi(n)^{2}}{n}=\frac{(p-1)^{2}(q-1)^{2}}{p}>4(p-1)^{2}
$$

and so $\bar{X}_{n}$ is not Ramanujan. As in previous cases, if $n=p^{2} q$, then $\bar{X}_{n}$ is not Ramanujan.

\section{References}

[1] A. Droll, A classification of ramanujan unitary cayley graphs, The Electronic Journal of Combinatorics, 29 (2010).

[2] C. Godsil and G. Royle, Algebraic graph theory, In: Algebraic Graph Theory, Springer, New York, 2001.

[3] A. Ilic, The energy of unitary cayley graphs, Linear Algebra Appl, 431 (2009), 1881-1889.

[4] W. Klotz and T. Sander, Some properties of unitary cayley graphs, The Electronic Journal of Combinatorics, 14 (2007).

[5] R. Murty, Ramanujan graphs, Journal of the Ramanujan Math, Society, 18 (2003), 1-20. 